\title{
QUASICONFORMAL AND LIPSCHITZ HARMONIC MAPPINGS OF THE UNIT DISK ONTO BOUNDED CONVEX DOMAINS
}

\author{
Dariusz Partyka and Ken-ichi Sakan
}

The John Paul II Catholic University of Lublin, Institute of Mathematics and Computer Science

Al. Racławickie 14, P.O. Box 129, 20-950 Lublin, Poland; partyka@kul.lublin.pl, and

The State School of Higher Education in Chełm, Institute of Mathematics

and Information Technology, Pocztowa 54, 22-100 Chełm, Poland

Osaka City University, Graduate School of Science, Department of Mathematics

Sugimoto, Sumiyoshi-ku, Osaka, 558, Japan; ksakan@sci.osaka-cu.ac.jp

\begin{abstract}
We study the Lipschitz property of a harmonic injective and sense-preserving mapping $F$ of the unit disk $\mathbf{D}$ onto a bounded convex domain $\Omega$ in the complex plane $\mathbf{C}$. In particular we show that $F$ is bi-Lipschitz iff $F$ is quasiconformal and Lipschitz. To this end we establish some auxiliary properties of harmonic mappings dealing with the boundary radial limiting values of the formal derivatives $\partial F$ and $\bar{\partial} F$.
\end{abstract}

\section{Introduction}

Let $\mathbf{D}(a, r):=\{z \in \mathbf{C}:|z-a|<r\}$ and $\mathbf{T}(a, r):=\{z \in \mathbf{C}:|z-a|=r\}$ for $a \in \mathbf{C}$ and $r>0$. In particular $\mathbf{D}:=\mathbf{D}(0,1)$ and $\mathbf{T}:=\mathbf{T}(0,1)$ are the unit disk and unit circle, respectively.

In 2002 Pavlović proved that for a harmonic injective and sense-preserving mapping $F: \mathbf{D} \rightarrow \mathbf{C}$ if $F(\mathbf{D})=\mathbf{D}$, then $F$ is quasiconformal iff $F$ is bi-Lipschitz; cf. [18] and also [15, Thm. 3.3] for a more quantitative form. This equivalence was extended by Kalaj in 2008 to the case where $F$ maps $\mathbf{D}$ onto a convex domain $\Omega$ in $\mathbf{C}$ bounded by a sufficiently smooth Jordan curve; cf. [9, Thm. 3.1 and Cor. 3.4] and also [11]. On the other hand, it is easily seen that a conformal mapping $F$ of $\mathbf{D}$ onto a rectangle has the unbounded derivative $F^{\prime}$, and so $F$ is not Lipschitz. Therefore Pavlović's equivalence is - in general - not valid in the case where $F(\mathbf{D})$ is a convex domain. This means that a harmonic injective and sense-preserving mapping $F: \mathbf{D} \rightarrow \mathbf{C}$ is biLipschitz provided $F$ is quasiconformal and some additional property is postulated. In this paper we show that for a harmonic injective and sense-preserving mapping $F: \mathbf{D} \rightarrow \mathbf{C}$ if $F(\mathbf{D})$ is a bounded convex domain, then $F$ is quasiconformal and Lipschitz iff $F$ is bi-Lipschitz; cf. Theorem 3.4. This theorem provides also three additional equivalent conditions. One of them gives a boundary description of such $F$; cf. the property (v) in Theorem 3.4. This property complements Kalaj's results [9, Thm. 3.1 and Cor. 3.4] as stated in Remark 4.2 and Corollary 4.3. The last corollary generalizes Pavlović's theorem from [18]. The equivalence (ii) $\Longleftrightarrow(v)$ in Theorem 3.4 also extends the authors result [12, Cor. 4.2].

doi:10.5186/aasfm.2014.3940

2010 Mathematics Subject Classification: Primary 30C55, 30C62.

Key words: Harmonic mappings, Lipschitz mappings, Poisson integral, Jacobian, quasiconformal mappings. 
The basic tool for our considerations is the following lemma being a development of the method from [13, pp. 25-26].

Lemma A. [16, Lem. 1.1] Given a harmonic function $u: \mathbf{D} \rightarrow \mathbf{R}$ and $a, b \in \mathbf{R}$ satisfying $a<b$, assume that $u(0)=0$ and

$$
a \leq u(z) \leq b, \quad z \in \mathbf{D} .
$$

Then

$$
u(z) \leq 2 \frac{b-a}{\pi} \arctan \frac{|z|+|p|}{1+|p||z|}+\frac{b+a}{2}, \quad z \in \mathbf{D}
$$

where

$$
p:=-\mathrm{i} \tan \frac{\pi}{4} \frac{b+a}{b-a} .
$$

In Section 1 we show some auxiliary properties of harmonic mappings dealing with the boundary radial limiting values of the formal derivatives $\partial F$ and $\bar{\partial} F$, where $F$ is a harmonic mapping given by the Poisson integral $\mathrm{P}[f]$ of a function $f: \mathbf{T} \rightarrow \mathbf{C}$ of bounded variation; cf. Lemma 1.1, Lemma 1.2 and Corollary 1.3. In Section 2 we use Lemma A and Lemma 1.1 to prove Lemma 2.3, which yields Lemma 2.4 and Theorem 2.5 providing lower bounds for the boundary radial limiting values of the Jacobian $\mathrm{J}[F]$. From Theorem 2.5 we infer Corollary 2.6 which gives lower bounds of the Jacobian $\mathrm{J}[F]$ in $\mathbf{D}$. Theorem 2.5 and Corollary 2.6 considerably extend Kalaj's results [8, Thm. 2.8 and Cor. 2.9] as pointed out in Remark 2.7. From Corollary 1.3 we infer in Section 3 useful estimations for the Lipschitz constant L $(F)$ of the mapping $F$ in terms of some quantities determined by the function $f$; cf. Lemma 3.1 and Theorem 3.2. Next we apply Theorem 2.5, Theorem 3.2 and [17, Thm. 3.8] to show Theorem 3.4, which is the main result in this paper. It extends the results by Zhu [21, Thm. 3] and by Kalaj [8, Thm. 3.2] in the sense pointed out in Remark 3.5. Section 4 contains applications of the results from the previous section. Corollary 4.1 extends Zhu's [21, Cor. 1]. Remark 4.2 and Corollary 4.3 complement Kalaj's results [9, Thm. 3.1 and Cor. 3.4].

\section{Boundary properties of the Poisson integral}

Given a function $f: \mathbf{T} \rightarrow \mathbf{C}$ and $z=\mathrm{e}^{\mathrm{i} \theta} \in \mathbf{T}$ we define

$$
\begin{aligned}
f^{\prime}(z) & :=\lim _{u \rightarrow z} \frac{f(u)-f(z)}{u-z}, \\
\dot{f}(z) & :=\lim _{t \rightarrow \theta} \frac{f\left(\mathrm{e}^{\mathrm{i} t}\right)-f\left(\mathrm{e}^{\mathrm{i} \theta}\right)}{t-\theta},
\end{aligned}
$$

provided the limits exist as well as $f^{\prime}(z):=0$ and $\dot{f}(z):=0$ otherwise. Obviously,

$$
\dot{f}(z)=\mathrm{i} z f^{\prime}(z) \text { and }|\dot{f}(z)|=\left|f^{\prime}(z)\right| \text {. }
$$

If $f$ is additionally integrable on $\mathbf{T}$, then we denote by $\mathrm{P}[f](z)$ the Poisson integral of $f$ at $z \in \mathbf{D}$, i.e.,

$$
\mathrm{P}[f](z):=\frac{1}{2 \pi} \int_{\mathbf{T}} f(u) \operatorname{Re} \frac{u+z}{u-z}|\mathrm{~d} u|, \quad z \in \mathbf{D} .
$$

Here and in the sequel integrable means integrable in the sense of Lebesgue. The Poisson integral $\mathrm{P}[f]$ is the unique solution to the Dirichlet problem for the unit 
disk $\mathbf{D}$ provided the boundary function $f$ is continuous; cf. e.g. [6, Thm. 2.11]. This means that $\mathrm{P}[f]$ is a harmonic mapping in $\mathbf{D}$ which has a continuous extension to the closed disk $\mathrm{cl}(\mathbf{D})$ and its boundary limiting valued function is identical with $f$. Here and subsequently, $\operatorname{cl}(A)$ stands for the closure of a set $A \subset \mathbf{C}$ in the Euclidian topology. If $f$ is a function of bounded variation, then we write $\mathrm{P}[\mathrm{d} f](z)$ for the Poisson-Stieltjes integral of $f$ at $z \in \mathbf{D}$, i.e.,

$$
\mathrm{P}[\mathrm{d} f](z):=\frac{1}{2 \pi} \int_{\mathbf{T}} \operatorname{Re} \frac{u+z}{u-z} \mathrm{~d} f(u), \quad z \in \mathbf{D} .
$$

We will use the standard notations $\partial:=\frac{1}{2}\left(\partial_{x}-\mathrm{i} \partial_{y}\right)$ and $\bar{\partial}:=\frac{1}{2}\left(\partial_{x}+\mathrm{i} \partial_{y}\right)$ for the so-called formal derivatives operators. Analyzing the proof of $[12$, Lem. 2.1] we state the following lemma.

Lemma 1.1. Let $f: \mathbf{T} \rightarrow \mathbf{C}$ be a function of bounded variation and differentiable at a point $z \in \mathbf{T}$. If the limit $\lim _{r \rightarrow 1^{-}} \frac{\mathrm{d}}{\mathrm{d} r} \mathrm{P}[f](r z)$ exists, then the remaining limits in (1.6), (1.7) and (1.8) exist and

$$
\begin{aligned}
& 2 z \lim _{r \rightarrow 1^{-}} \partial \mathrm{P}[f](r z)=\lim _{r \rightarrow 1^{-}} \frac{f(z)-\mathrm{P}[f](r z)}{1-r}+z f^{\prime}(z), \\
& 2 \bar{z} \lim _{r \rightarrow 1^{-}} \bar{\partial} \mathrm{P}[f](r z)=\lim _{r \rightarrow 1^{-}} \frac{f(z)-\mathrm{P}[f](r z)}{1-r}-z f^{\prime}(z)
\end{aligned}
$$

as well as

$$
\lim _{r \rightarrow 1^{-}} \frac{\mathrm{d}}{\mathrm{d} r} \mathrm{P}[f](r z)=\lim _{r \rightarrow 1^{-}} \frac{f(z)-\mathrm{P}[f](r z)}{1-r} .
$$

Proof. Given $z \in \mathbf{T}$ assume that a function $f$ is of bounded variation and differentiable at $z$ and the first limit in (1.8) exists. From (1.4) it follows that for every $r \in(0 ; 1)$,

$$
\begin{aligned}
\partial \mathrm{P}[f](r z) & =\frac{1}{2 \pi} \int_{\mathbf{T}} f(u) \frac{u}{(u-r z)^{2}}|\mathrm{~d} u| \\
& =-\frac{1}{2 \pi} \frac{1}{2 \mathrm{i} r z} \int_{0}^{2 \pi} f\left(\mathrm{e}^{\mathrm{i} t}\right) \frac{-2 \mathrm{i} r z \mathrm{e}^{\mathrm{i} t}}{\left(\mathrm{e}^{\mathrm{i} t}-r z\right)^{2}} \mathrm{~d} t \\
& =-\frac{1}{2 \pi} \frac{1}{2 \mathrm{i} r z} \int_{0}^{2 \pi} f\left(\mathrm{e}^{\mathrm{i} t}\right) \frac{\mathrm{d}}{\mathrm{d} t}\left(\frac{\mathrm{e}^{\mathrm{i} t}+r z}{\mathrm{e}^{\mathrm{i} t}-r z}\right) \mathrm{d} t
\end{aligned}
$$

and consequently,

$$
\begin{aligned}
\overline{\overline{\mathrm{P}}[f](r z)} & =\partial \overline{\mathrm{P}[f]}(r z)=\partial \mathrm{P}[\bar{f}](r z) \\
& =-\frac{1}{2 \pi} \frac{1}{2 \mathrm{i} r z} \int_{0}^{2 \pi} \overline{f\left(\mathrm{e}^{\mathrm{i} t}\right)} \frac{\mathrm{d}}{\mathrm{d} t}\left(\frac{\mathrm{e}^{\mathrm{i} t}+r z}{\mathrm{e}^{\mathrm{i} t}-r z}\right) \mathrm{d} t .
\end{aligned}
$$

Since $f$ is a function of bounded variation, we may integrate by parts the last integrals in (1.9) and (1.10). As a result we obtain

$$
\begin{aligned}
& r z \partial \mathrm{P}[f](r z)=\frac{1}{4 \pi \mathrm{i}} \int_{0}^{2 \pi} \frac{\mathrm{e}^{\mathrm{i} t}+r z}{\mathrm{e}^{\mathrm{i} t}-r z} \mathrm{~d} f\left(\mathrm{e}^{\mathrm{i} t}\right) \\
& r z \overline{\bar{\partial} \mathrm{P}[f](r z)}=\frac{1}{4 \pi \mathrm{i}} \int_{0}^{2 \pi} \frac{\mathrm{e}^{\mathrm{i} t}+r z}{\mathrm{e}^{\mathrm{i} t}-r z} \mathrm{~d} \overline{f\left(\mathrm{e}^{\mathrm{i} t}\right)} .
\end{aligned}
$$


By (1.11) we have

$$
r z \partial \mathrm{P}[f](r z)-r \bar{z} \bar{\partial} \mathrm{P}[f](r z)=\frac{1}{2 \pi \mathrm{i}} \int_{0}^{2 \pi} \operatorname{Re}\left(\frac{\mathrm{e}^{\mathrm{i} t}+r z}{\mathrm{e}^{\mathrm{i} t}-r z}\right) \mathrm{d} f\left(\mathrm{e}^{\mathrm{i} t}\right)=\frac{1}{\mathrm{i}} \mathrm{P}[\mathrm{d} f](r z) .
$$

By (1.2) we see that

$$
\lim _{r \rightarrow 1^{-}}(r z \partial \mathrm{P}[f](r z)-r \bar{z} \bar{\partial} \mathrm{P}[f](r z))=\lim _{r \rightarrow 1^{-}} \frac{1}{\mathrm{i}} \mathrm{P}[\mathrm{d} f](r z)=\frac{1}{\mathrm{i}} \dot{f}(z)=z f^{\prime}(z) ;
$$

cf. e.g. [2, Thm. 1.2] or [20, Thm. 11.12]. Since the $\operatorname{limit} \lim _{r \rightarrow 1^{-}} \frac{\mathrm{d}}{\mathrm{d} r} \mathrm{P}[f](r z)$ exists, we conclude from (1.13) and

$$
\frac{\mathrm{d}}{\mathrm{d} r} \mathrm{P}[f](r z)=z \partial \mathrm{P}[f](r z)+\bar{z} \bar{\partial} \mathrm{P}[f](r z), \quad 0 \leq r<1,
$$

that the following limits exist and

$$
\begin{aligned}
& 2 z \lim _{r \rightarrow 1^{-}} \partial \mathrm{P}[f](r z)=\lim _{r \rightarrow 1^{-}} \frac{\mathrm{d}}{\mathrm{d} r} \mathrm{P}[f](r z)+z f^{\prime}(z), \\
& 2 \bar{z} \lim _{r \rightarrow 1^{-}} \bar{\partial} \mathrm{P}[f](r z)=\lim _{r \rightarrow 1^{-}} \frac{\mathrm{d}}{\mathrm{d} r} \mathrm{P}[f](r z)-z f^{\prime}(z) .
\end{aligned}
$$

Furthermore, the following limit exists and

$$
\lim _{r \rightarrow 1^{-}} \frac{1}{1-r} \int_{r}^{1} \frac{\mathrm{d}}{\mathrm{d} t} \mathrm{P}[f](t z) \mathrm{d} t=\lim _{r \rightarrow 1^{-}} \frac{\mathrm{d}}{\mathrm{d} r} \mathrm{P}[f](r z) .
$$

Hence and by the equality

$$
\frac{f(z)-\mathrm{P}[f](r z)}{1-r}=\frac{1}{1-r} \int_{r}^{1} \frac{\mathrm{d}}{\mathrm{d} t} \mathrm{P}[f](t z) \mathrm{d} t, \quad r \in[0 ; 1)
$$

we see that the second limit in (1.8) exists and the equality (1.8) holds. Therefore all the limits in (1.6) and (1.7) exist. Combining the equalities (1.15) and (1.8) we derive the equalities (1.6) and (1.7), which completes the proof.

We recall that the harmonic conjugate operator $\mathrm{A}$ is defined for a function $f: \mathbf{T} \rightarrow$ $\mathbf{C}$ integrable on $\mathbf{T}$ and $z \in \mathbf{T}$ as follows:

$$
\mathrm{A}[f](z):=\frac{1}{2 \pi} \lim _{r \rightarrow 1^{-}} \int_{0}^{2 \pi} f\left(\mathrm{e}^{\mathrm{i} t}\right) \operatorname{Im} \frac{\mathrm{e}^{\mathrm{i} t}+r z}{\mathrm{e}^{\mathrm{i} t}-r z} \mathrm{~d} t,
$$

whenever the limit exists and $\mathrm{A}[f](z):=0$ otherwise. It is known that for a.e. $z \in \mathbf{T}$ the limit exists; cf. [5, Chap. III, Lem. 1.1]. If $f$ is real valued, then the function

$$
\mathbf{D} \ni z \mapsto \frac{1}{2 \pi} \int_{0}^{2 \pi} f\left(\mathrm{e}^{\mathrm{i} t}\right) \operatorname{Im} \frac{\mathrm{e}^{\mathrm{i} t}+z}{\mathrm{e}^{\mathrm{i} t}-z} \mathrm{~d} t
$$

is the harmonic conjugate function of $\mathrm{P}[f]$, which justifies the name of the operator A. Replacing $\mathrm{P}[f]$ by the Poisson-Stieltjes integral $\mathrm{P}[\mathrm{d} f]$ we may define the harmonic conjugate operator A for every function $f: \mathbf{T} \rightarrow \mathbf{C}$ of bounded variation and $z \in \mathbf{T}$ as follows:

$$
\mathrm{A}[\mathrm{d} f](z):=\frac{1}{2 \pi} \lim _{r \rightarrow 1^{-}} \int_{0}^{2 \pi} \operatorname{Im} \frac{\mathrm{e}^{\mathrm{i} t}+r z}{\mathrm{e}^{\mathrm{i} t}-r z} \mathrm{~d} f\left(\mathrm{e}^{\mathrm{i} t}\right),
$$

whenever the limit exists and $\mathrm{A}[\mathrm{d} f](z):=0$ otherwise. Note that

$$
\mathrm{A}[\mathrm{d} f](z)=\mathrm{A}[\dot{f}](z) \quad \text { for a.e. } z \in \mathbf{T} \text {, }
$$


Quasiconformal and Lipschitz harmonic mappings of the unit disk onto bounded convex domains 815 provided $f$ is an absolutely continuous function.

Lemma 1.2. If $f: \mathbf{T} \rightarrow \mathbf{C}$ is a function of bounded variation, then for every $z \in \mathbf{T}$ the $\operatorname{limit}_{\lim _{r \rightarrow 1^{-}}} \frac{\mathrm{d}}{\mathrm{d} r} \mathrm{P}[f](r z)$ exists iff the limit in (1.17) exists, and the equality

$$
\lim _{r \rightarrow 1^{-}} \frac{\mathrm{d}}{\mathrm{d} r} \mathrm{P}[f](r z)=\mathrm{A}[\mathrm{d} f](z)
$$

holds provided one of the limits exists.

Proof. Given a function $f$ of bounded variation on $\mathbf{T}$ and $z \in \mathbf{T}$ we see, integrating by parts, that for every $r \in(0 ; 1)$,

$$
\begin{aligned}
2 \pi \frac{\mathrm{d}}{\mathrm{d} r} \mathrm{P}[f](r z) & =\frac{\mathrm{d}}{\mathrm{d} r} \int_{0}^{2 \pi} f\left(\mathrm{e}^{\mathrm{i} t}\right) \operatorname{Re} \frac{\mathrm{e}^{\mathrm{i} t}+r z}{\mathrm{e}^{\mathrm{i} t}-r z} \mathrm{~d} t=\int_{0}^{2 \pi} f\left(\mathrm{e}^{\mathrm{i} t}\right) \operatorname{Re} \frac{\mathrm{d}}{\mathrm{d} r} \frac{\mathrm{e}^{\mathrm{i} t}+r z}{\mathrm{e}^{\mathrm{i} t}-r z} \mathrm{~d} t \\
& =\int_{0}^{2 \pi} f\left(\mathrm{e}^{\mathrm{i} t}\right) \operatorname{Re} \frac{2 \mathrm{e}^{\mathrm{i} t} z}{\left(\mathrm{e}^{\mathrm{i} t}-r z\right)^{2}} \mathrm{~d} t=\int_{0}^{2 \pi} f\left(\mathrm{e}^{\mathrm{i} t}\right) \operatorname{Re} \frac{\mathrm{i}}{r} \frac{\mathrm{d}}{\mathrm{d} t} \frac{\mathrm{e}^{\mathrm{i} t}+r z}{\mathrm{e}^{\mathrm{i} t}-r z} \mathrm{~d} t \\
& =-\frac{1}{r} \int_{0}^{2 \pi} f\left(\mathrm{e}^{\mathrm{i} t}\right) \frac{\mathrm{d}}{\mathrm{d} t} \operatorname{Im} \frac{\mathrm{e}^{\mathrm{i} t}+r z}{\mathrm{e}^{\mathrm{i} t}-r z} \mathrm{~d} t=\frac{1}{r} \int_{0}^{2 \pi} \operatorname{Im} \frac{\mathrm{e}^{\mathrm{i} t}+r z}{\mathrm{e}^{\mathrm{i} t}-r z} \mathrm{~d} f\left(\mathrm{e}^{\mathrm{i} t}\right) .
\end{aligned}
$$

Hence and by (1.17) we see that both the limits in (1.19) and (1.17) simultaneously exist or not, and the equality

$$
\lim _{r \rightarrow 1^{-}} \frac{\mathrm{d}}{\mathrm{d} r} \mathrm{P}[f](r z)=\lim _{r \rightarrow 1^{-}} r \frac{\mathrm{d}}{\mathrm{d} r} \mathrm{P}[f](r z)=\mathrm{A}[\mathrm{d} f](z)
$$

holds in the first case, which is our claim.

For $p>0$ let $\mathrm{H}^{p}(\mathbf{D})$ stand for the Hardy space of holomorphic functions in the unit disk; cf. e.g. [2, Sect. 1.1].

Corollary 1.3. If $f: \mathbf{T} \rightarrow \mathbf{C}$ is a function of bounded variation, then the derivative functions $\partial \mathrm{P}[f], \bar{\partial} \mathrm{P}[f] \in \mathrm{H}^{p}(\mathbf{D})$ for $p \in(0 ; 1)$ and for a.e. $z \in \mathbf{T}$ the following limits exist and the equalities hold

$$
\begin{aligned}
\mathrm{A}[\mathrm{d} f](z) & =\lim _{r \rightarrow 1^{-}} \frac{\mathrm{d}}{\mathrm{d} r} \mathrm{P}[f](r z)=\lim _{r \rightarrow 1^{-}} \frac{f(z)-\mathrm{P}[f](r z)}{1-r} \\
& =\lim _{r \rightarrow 1^{-}}(z \partial \mathrm{P}[f](r z)+\bar{z} \bar{\partial} \mathrm{P}[f](r z))
\end{aligned}
$$

as well as

$$
\begin{aligned}
\lim _{r \rightarrow 1^{-}} \partial \mathrm{P}[f](r z) & =\frac{\bar{z}}{2}\left(\mathrm{~A}[\mathrm{~d} f](z)+z f^{\prime}(z)\right), \\
\lim _{r \rightarrow 1^{-}} \bar{\partial} \mathrm{P}[f](r z) & =\frac{z}{2}\left(\mathrm{~A}[\mathrm{~d} f](z)-z f^{\prime}(z)\right) .
\end{aligned}
$$

Proof. Assume that $f$ is a function of bounded variation on $\mathbf{T}$. Then $\bar{f}$ is a function of bounded variation on $\mathbf{T}$ with the same variation as $f$. From the equalities (1.11) it follows that for every $z \in \mathbf{D}$,

$$
\begin{aligned}
& z \partial \mathrm{P}[f](z)=\frac{1}{4 \pi \mathrm{i}} \int_{0}^{2 \pi} \frac{\mathrm{e}^{\mathrm{i} t}+z}{\mathrm{e}^{\mathrm{i} t}-z} \mathrm{~d} f\left(\mathrm{e}^{\mathrm{i} t}\right)=\frac{1}{2 \pi \mathrm{i}} \int_{0}^{2 \pi} \frac{\mathrm{e}^{\mathrm{i} t}}{\mathrm{e}^{\mathrm{i} t}-z} \mathrm{~d} f\left(\mathrm{e}^{\mathrm{i} t}\right) \\
& z \overline{\bar{\partial} \mathrm{P}[f](z)}=\frac{1}{4 \pi \mathrm{i}} \int_{0}^{2 \pi} \frac{\mathrm{e}^{\mathrm{i} t}+z}{\mathrm{e}^{\mathrm{i} t}-z} \mathrm{~d} \overline{f\left(\mathrm{e}^{\mathrm{i} t}\right)}=\frac{1}{2 \pi \mathrm{i}} \int_{0}^{2 \pi} \frac{\mathrm{e}^{\mathrm{i} t}}{\mathrm{e}^{\mathrm{i} t}-z} \mathrm{~d} \overline{f\left(\mathrm{e}^{\mathrm{i} t}\right)} .
\end{aligned}
$$


Applying now the Smirnov theorem we conclude that $\partial \mathrm{P}[f] \in \mathrm{H}^{p}(\mathbf{D})$ and $\overline{\bar{\partial} \mathrm{P}[f]} \in$ $\mathrm{H}^{p}(\mathbf{D})$ for $p \in(0 ; 1)$; cf. [2, Thm. 3.5] and [19, p. 65]. Therefore the functions $\partial \mathrm{P}[f]$ and $\bar{\partial} \mathrm{P}[f]$ have radial limits a.e. on T; cf. [2, Thm. 2.2]. Hence and by (1.14) the limit $\lim _{r \rightarrow 1^{-}} \frac{\mathrm{d}}{\mathrm{d} r} \mathrm{P}[f](r z)$ exists for a.e. $z \in \mathbf{T}$ and

$$
\lim _{r \rightarrow 1^{-}} \frac{\mathrm{d}}{\mathrm{d} r} \mathrm{P}[f](r z)=\lim _{r \rightarrow 1^{-}}(z \partial \mathrm{P}[f](r z)+\bar{z} \bar{\partial} \mathrm{P}[f](r z)) .
$$

From Lemmas 1.1 and 1.2 it follows that the equalities (1.20) hold for a.e. $z \in \mathbf{T}$. Combining (1.20) with (1.6) and (1.7) we obtain for a.e. $z \in \mathbf{T}$ the equalities (1.21) and (1.22) respectively, which completes the proof.

\section{Boundary behaviour of the Jacobian}

Definition 2.1. We say that a point $v \in \mathbf{C}$ is linearly accessible from outside of a set $\Omega \subset \mathbf{C}$ if there exists $\zeta \in \mathbf{T}$ such that

$$
\operatorname{Re}(\zeta w) \leq \operatorname{Re}(\zeta v), \quad w \in \Omega .
$$

Remark 2.2. The condition (2.1) means geometrically that there exists a closed half-plane $H$ such that its boundary line passes through the point $v$ and $\Omega \subset H$. In particular, if $\Omega$ is a convex domain in $\mathbf{C}, \Omega \neq \mathbf{C}$, then each point $v \in \mathbf{C} \backslash \Omega$ is linearly accessible from outside of $\Omega$. Indeed, suppose first that $v \notin \mathrm{cl}(\Omega)$. It is easy to check that $\operatorname{cl}(\Omega)$ is a convex set. Since $\operatorname{cl}(\Omega)$ is also closed, there exists $v^{\prime} \in \operatorname{cl}(\Omega)$ such that

$$
0 \neq\left|v^{\prime}-v\right| \leq|z-v|, \quad z \in \operatorname{cl}(\Omega) .
$$

Given $z \in \operatorname{cl}(\Omega)$ we see that $(1-t) v^{\prime}+t z \in \operatorname{cl}(\Omega)$ for $t \in[0 ; 1]$. Hence and by $(2.2)$ we see that for every $t \in(0 ; 1]$,

$$
\left|v^{\prime}-v\right|^{2} \leq\left|(1-t) v^{\prime}+t z-v\right|^{2}=\left|v^{\prime}-v\right|^{2}-2 t \operatorname{Re}\left[\left(v^{\prime}-v\right) \overline{\left(v^{\prime}-z\right)}\right]+t^{2}\left|v^{\prime}-z\right|^{2},
$$

and consequently

$$
\operatorname{Re}\left[\left(v-v^{\prime}\right) \overline{\left(z-v^{\prime}\right)}\right] \leq \frac{t}{2}\left|v^{\prime}-z\right|^{2} \rightarrow 0 \quad \text { as } t \rightarrow 0^{+} .
$$

Therefore $\operatorname{Re}\left[\left(v-v^{\prime} \overline{\left(z-v^{\prime}\right)}\right] \leq 0\right.$, and setting $\zeta:=\overline{\left(v-v^{\prime}\right)} /\left|v-v^{\prime}\right|$ we see that $\zeta \in \mathbf{T}, \operatorname{Re}\left[\left(z-v^{\prime}\right) \zeta\right] \leq 0$ and

$$
\operatorname{Re}[v \zeta]-\operatorname{Re}\left[v^{\prime} \zeta\right]=\operatorname{Re}\left[\left(v-v^{\prime}\right) \zeta\right]=\left|v-v^{\prime}\right|>0
$$

Combining these inequalities we obtain

$$
\operatorname{Re}[z \zeta] \leq \operatorname{Re}\left[v^{\prime} \zeta\right]<\operatorname{Re}[v \zeta], \quad z \in \operatorname{cl}(\Omega),
$$

and so the condition (2.1) holds in the case where $v \in \mathbf{C} \backslash \operatorname{cl}(\Omega)$. Suppose now that $v \in \operatorname{cl}(\Omega) \backslash \Omega$. Choosing arbitrarily $z_{0} \in \Omega$ we see that $v_{n}:=(1+1 / n)\left(v-z_{0}\right)+z_{0} \notin$ $\operatorname{cl}(\Omega)$ for $n \in \mathbf{N}$. Then there exists a sequence $\mathbf{N} \ni n \mapsto \zeta_{n} \in \mathbf{T}$ such that

$$
\operatorname{Re}\left[z \zeta_{n}\right]<\operatorname{Re}\left[v_{n} \zeta_{n}\right], \quad z \in \operatorname{cl}(\Omega), n \in \mathbf{N} .
$$

Since $\mathbf{T}$ is a compact set, there exists a subsequence $\mathbf{N} \ni k \mapsto \zeta_{n_{k}} \in \mathbf{T}$ and $\zeta \in \mathbf{T}$ such that $\zeta_{n_{k}} \rightarrow \zeta$ as $k \rightarrow \infty$. A passage to the limit in (2.3) implies that

$$
\operatorname{Re}[z \zeta]=\lim _{k \rightarrow \infty} \operatorname{Re}\left[z \zeta_{n_{k}}\right] \leq \lim _{k \rightarrow \infty} \operatorname{Re}\left[v_{n_{k}} \zeta_{n_{k}}\right]=\operatorname{Re}[v \zeta], \quad z \in \operatorname{cl}(\Omega)
$$

and so the condition (2.1) holds in the case where $v \in \operatorname{cl}(\Omega) \backslash \Omega$. Therefore each $v \in \mathbf{C} \backslash \Omega$ is linearly accessible from outside of $\Omega$, as claimed. 
Let $\mathrm{J}[F]$ stand for the Jacobian of a differentiable mapping $F: \mathbf{D} \rightarrow \mathbf{C}$, i.e.,

$$
\mathrm{J}[F](z):=|\partial F(z)|^{2}-|\bar{\partial} F(z)|^{2}, \quad z \in \mathbf{D} .
$$

Lemma 2.3. Given a harmonic mapping $F: \mathbf{D} \rightarrow \mathbf{C}$ assume that $F(0)=0 \neq$ $\mathrm{J}[F](0)$. If $v \in \mathbf{C}$ is a point linearly accessible from outside of $\Omega:=F(\mathbf{D})$ and $\zeta \in \mathbf{T}$ satisfies the condition (2.1), then for every $z \in \mathbf{T}$,

$$
\liminf _{r \rightarrow 1^{-}} \operatorname{Re} \frac{\zeta v-\zeta F(r z)}{1-r} \geq \frac{a+b}{\pi} \tan \left(\frac{\pi}{2} \frac{\min (a, b)}{a+b}\right) \geq \frac{\min (a, b)}{2},
$$

where

$$
a:=-\inf _{u \in \mathbf{D}} \operatorname{Re}(\zeta F(u)) \quad \text { and } \quad b:=\sup _{u \in \mathbf{D}} \operatorname{Re}(\zeta F(u)) .
$$

Proof. Fix $F: \mathbf{D} \rightarrow \mathbf{C}$ and $\zeta \in \mathbf{T}$ satisfying the assumptions. Then $F_{\zeta}:=\zeta F$ is a harmonic mapping on $\mathbf{D}$ and $F_{\zeta}(0)=0$. Since $\mathrm{J}[F](0) \neq 0=F(0)$ we see that 0 is an inner point of $\Omega$. Hence and by (2.6) it follows that $-a<0<b$ and

$$
-a \leq \operatorname{Re}\left(F_{\zeta}(u)\right) \leq b, \quad u \in \mathbf{D} .
$$

Applying now Lemma A we see that for arbitrarily fixed $z \in \mathbf{T}$,

$$
\operatorname{Re} F_{\zeta}(r z) \leq 2 \frac{b+a}{\pi} \arctan \frac{r+|p|}{1+|p| r}+\frac{b-a}{2}, \quad r \in[0 ; 1),
$$

where

$$
p:=-\mathrm{i} \tan \frac{\pi}{4} \frac{b-a}{b+a} .
$$

Since $v$ is a point linearly accessible from outside of $\Omega$, we conclude from $(2.1),(2.6)$ and (2.7) that for every $r \in[0 ; 1)$,

$$
\begin{aligned}
\operatorname{Re}\left(\zeta v-F_{\zeta}(r z)\right) & \geq b-\operatorname{Re} F_{\zeta}(r z) \geq b-2 \frac{b+a}{\pi} \arctan \frac{r+|p|}{1+|p| r}-\frac{b-a}{2} \\
& =\frac{b+a}{2}\left(1-\frac{4}{\pi} \arctan \frac{r+|p|}{1+|p| r}\right) \\
& =\frac{2(b+a)}{\pi}\left(\frac{\pi}{4}-\arctan \frac{r+|p|}{1+|p| r}\right) .
\end{aligned}
$$

Hence

$$
\liminf _{r \rightarrow 1^{-}} \operatorname{Re} \frac{\zeta v-\zeta F(r z)}{1-r} \geq \liminf _{r \rightarrow 1^{-}} \frac{2(b+a)}{\pi(1-r)}\left(\frac{\pi}{4}-\arctan \frac{r+|p|}{1+|p| r}\right) .
$$

Since

$$
\frac{\mathrm{d}}{\mathrm{d} r} \arctan \frac{r+|p|}{1+|p| r}=\frac{1}{2} \frac{1-|p|}{1+|p|},
$$

we conclude from (2.9) that

$$
\begin{aligned}
\liminf _{r \rightarrow 1^{-}} \operatorname{Re} \frac{\zeta v-\zeta F(r z)}{1-r} & \geq \frac{2(b+a)}{\pi} \lim _{r \rightarrow 1^{-}} \frac{1}{1-r}\left(\frac{\pi}{4}-\arctan \frac{r+|p|}{1+|p| r}\right) \\
& =\frac{b+a}{\pi} \frac{1-|p|}{1+|p|} .
\end{aligned}
$$


Setting $\alpha:=\frac{\pi}{4} \frac{b-a}{b+a}$ we deduce from (2.8) that

$$
\begin{aligned}
\frac{1-|p|}{1+|p|} & =\frac{1-\tan |\alpha|}{1+\tan |\alpha|}=\frac{\cos |\alpha|-\sin |\alpha|}{\cos |\alpha|+\sin |\alpha|}=\frac{\sin \left(\frac{\pi}{4}-|\alpha|\right)}{\cos \left(\frac{\pi}{4}-|\alpha|\right)}=\tan \left(\frac{\pi}{4}-|\alpha|\right) \\
& =\tan \left(\frac{\pi}{4}\left(1-\frac{|b-a|}{b+a}\right)\right)=\tan \left(\frac{\pi}{4} \frac{b+a-|b-a|}{b+a}\right) \\
& =\tan \left(\frac{\pi}{2} \frac{\min (b, a)}{b+a}\right) \geq \frac{\pi}{2} \frac{\min (b, a)}{b+a}
\end{aligned}
$$

because $\tan t \geq t$ for $t \in[0 ; \pi / 2)$. Combining this with (2.10) we derive the inequalities (2.5), which completes the proof.

Lemma 2.4. Let $f: \mathbf{T} \rightarrow \mathbf{C}$ be a function of bounded variation and differentiable at a point $z \in \mathbf{T}$ such that $\mathrm{P}[f](0)=0 \neq \mathrm{J}[\mathrm{P}[f]](0)$, the $\operatorname{limit} \lim _{r \rightarrow 1^{-}} \frac{\mathrm{d}}{\mathrm{d} r} \mathrm{P}[f](r z)$ exists and

$$
\liminf _{r \rightarrow 1^{-}} \mathrm{J}[\mathrm{P}[f]](r z) \geq 0
$$

If $v:=f(z)$ is a point linearly accessible from outside of $\Omega:=\mathrm{P}[f](\mathbf{D})$ and $\zeta \in \mathbf{T}$ satisfies the condition (2.1), then the following limits exist and

$$
\begin{aligned}
\lim _{r \rightarrow 1^{-}} \mathrm{J}[\mathrm{P}[f]](r z) & =\left|f^{\prime}(z)\right| \lim _{r \rightarrow 1^{-}} \operatorname{Re} \frac{\zeta f(z)-\zeta \mathrm{P}[f](r z)}{1-r} \\
& \geq\left|f^{\prime}(z)\right| \frac{a+b}{\pi} \tan \left(\frac{\pi}{2} \frac{\min (a, b)}{a+b}\right) \geq\left|f^{\prime}(z)\right| \frac{\min (a, b)}{2},
\end{aligned}
$$

where

$$
a:=-\inf _{u \in \mathbf{D}} \operatorname{Re}(\zeta \mathrm{P}[f](u)) \quad \text { and } \quad b:=\sup _{u \in \mathbf{D}} \operatorname{Re}(\zeta \mathrm{P}[f](u)) .
$$

Proof. Fix $f: \mathbf{T} \rightarrow \mathbf{C}$ and $z, \zeta \in \mathbf{T}$ satisfying the assumptions. Then $F_{\zeta}:=\zeta \mathrm{P}[f]$ is a harmonic mapping in $\mathbf{D}$ and $F_{\zeta}(0)=0$. Since $\mathrm{J}\left[F_{\zeta}\right](0) \neq 0$ we see that 0 is an inner point of $F_{\zeta}(\mathbf{D})$. Hence and by (2.13) it follows that $-a<0<b$ and

$$
-a \leq \operatorname{Re}\left(F_{\zeta}(u)\right) \leq b \leq \operatorname{Re}(\zeta f(z)), \quad u \in \mathbf{D} .
$$

The function $f$ is bounded as a function of bounded variation. Therefore there

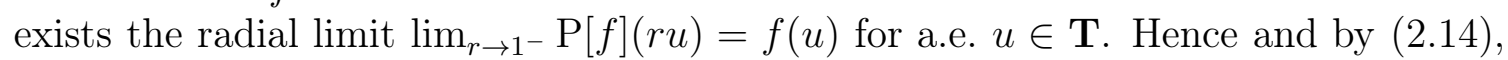
$\operatorname{Re}\left(\zeta f\left(\mathrm{e}^{\mathrm{i} t}\right)\right) \leq \operatorname{Re}\left(\zeta f\left(\mathrm{e}^{\mathrm{i} \theta}\right)\right)$ for a.e. $t \in \mathbf{R}$, where $z=\mathrm{e}^{\mathrm{i} \theta}$. Since the function $f$ is differentiable at $z$ we have

$$
0=\frac{\mathrm{d}}{\mathrm{d} t} \operatorname{Re}\left(\zeta f\left(\mathrm{e}^{\mathrm{i} t}\right)_{\mid t=\theta}=\operatorname{Re}\left(\mathrm{i} \zeta z f^{\prime}(z)\right)\right.
$$

and consequently

$$
\operatorname{Im}\left(\zeta z f^{\prime}(z)\right)=0
$$


From (1.6) and (1.7) of Lemma 1.1 it follows that the mapping $F:=\mathrm{P}[f]$ satisfies the following equalities

$$
\begin{aligned}
\lim _{r \rightarrow 1^{-}}\left(|\partial F(r z)|^{2}-|\bar{\partial} F(r z)|^{2}\right) & =\lim _{r \rightarrow 1^{-}} \operatorname{Re}\left(\overline{z f^{\prime}(z)} \frac{f(z)-F(r z)}{1-r}\right) \\
& =\lim _{r \rightarrow 1^{-}} \operatorname{Re}\left(\overline{\zeta z f^{\prime}(z)} \frac{\zeta f(z)-\zeta F(r z)}{1-r}\right) \\
& =\zeta z f^{\prime}(z) \lim _{r \rightarrow 1^{-}} \operatorname{Re} \frac{\zeta f(z)-\zeta F(r z)}{1-r} .
\end{aligned}
$$

Hence and by (2.11) we obtain

$$
0 \leq \liminf _{r \rightarrow 1^{-}} \mathrm{J}[F](r z)=\lim _{r \rightarrow 1^{-}} \mathrm{J}[F](r z)=\zeta z f^{\prime}(z) \lim _{r \rightarrow 1^{-}} \operatorname{Re} \frac{\zeta f(z)-\zeta F(r z)}{1-r} .
$$

From Lemma 2.3 it follows that

$$
\lim _{r \rightarrow 1^{-}} \operatorname{Re} \frac{\zeta f(z)-\zeta F(r z)}{1-r}=\liminf _{r \rightarrow 1^{-}} \operatorname{Re} \frac{\zeta f(z)-\zeta F(r z)}{1-r} \geq \frac{\min (a, b)}{2}>0 .
$$

Combining this with $(2.16)$ we see that $\zeta z f^{\prime}(z) \geq 0$, and so $\zeta z f^{\prime}(z)=\left|f^{\prime}(z)\right|$. Hence and by (2.16) we deduce the equality in (2.12). Applying now Lemma 2.3 once more we derive from (2.13) the inequalities in (2.12), which completes the proof.

Theorem 2.5. Let $f: \mathbf{T} \rightarrow \mathbf{C}$ be a function of bounded variation and differentiable at a point $z \in \mathbf{T}$ such that $\mathrm{P}[f](0)=0 \neq \mathrm{J}[\mathrm{P}[f]](0)$, the $\operatorname{limit}_{\lim _{r \rightarrow 1^{-}}} \frac{\mathrm{d}}{\mathrm{d} r} \mathrm{P}[f](r z)$ exists and the inequality (2.11) holds. If $f(z)$ is a point linearly accessible from outside of $\mathrm{P}[f](\mathbf{D})$, then the following limit exists and

$$
\lim _{r \rightarrow 1^{-}} \mathrm{J}[\mathrm{P}[f]](r z) \geq\left|f^{\prime}(z)\right| \frac{R_{1}+R_{2}}{\pi} \tan \left(\frac{\pi}{2} \frac{R_{1}}{R_{1}+R_{2}}\right) \geq\left|f^{\prime}(z)\right| \frac{R_{1}}{2}
$$

for all $R_{1}, R_{2}>0$ satisfying the condition

$$
\mathbf{D}\left(0, R_{1}\right) \subset \mathrm{P}[f](\mathbf{D}) \subset \mathbf{D}\left(0, R_{2}\right) .
$$

Proof. Fix $R_{1}, R_{2}>0$ satisfying the condition (2.18). Since $f(z)$ is a point linearly accessible from outside of $\Omega:=\mathrm{P}[f](\mathbf{D})$, there exists $\zeta \in \mathbf{T}$ satisfying the condition (2.1). From Lemma 2.4 it follows that (2.12) holds. By (2.13) and (2.18) we have $R_{1} \leq a \leq R_{2}$ and $R_{1} \leq b \leq R_{2}$. Hence $\max (a / b, b / a) \leq R_{2} / R_{1}$. Since $(0 ; \pi / 2) \ni$ $t \mapsto t^{-1} \tan t$ is an increasing function, we derive from (2.12) the inequalities (2.17), which is our assertion.

Corollary 2.6. Let $f$ be a sense-preserving homeomorphism of $\mathbf{T}$ onto the boundary $\Gamma$ of a bounded convex domain $\Omega$ in $\mathbf{C}$. If $\mathrm{P}[f](0)=0$, then for all $R_{1}, R_{2}>0$ satisfying the condition (2.18),

$$
\mathrm{J}[\mathrm{P}[f]](z) \geq d_{f} \frac{R_{1}+R_{2}}{\pi} \tan \left(\frac{\pi}{2} \frac{R_{1}}{R_{1}+R_{2}}\right) \geq d_{f} \frac{R_{1}}{2}, \quad z \in \mathbf{D},
$$

where

$$
d_{f}:=\underset{z \in \mathbf{T}}{\operatorname{essinf}}|\dot{f}(z)|
$$


Proof. Given $f$ satisfying the assumptions we see that $\Gamma$ is a rectifiable Jordan curve $([4])$, and thus $f$ is a function of bounded variation. By Radó-Kneser-Choquet Theorem $F:=\mathrm{P}[f]$ is a sense-preserving injective harmonic mapping of $\mathbf{D}$ onto $\Omega$; cf. [3, Sect. 3.1]. Furthermore, by Remark 2.2 for every $z \in \mathbf{T}, f(z)$ is a point linearly accessible from outside of $\Omega$. Fix $R_{1}, R_{2}>0$ satisfying the condition (2.18). From Theorem 2.5 and Corollary 1.3 it follows that the inequalities (2.17) hold for a.e. $z \in \mathbf{T}$, and so

$$
\lim _{r \rightarrow 1^{-}} \mathrm{J}[F](r z) \geq \lambda:=d_{f} \frac{R_{1}+R_{2}}{\pi} \tan \left(\frac{\pi}{2} \frac{R_{1}}{R_{1}+R_{2}}\right) \quad \text { for a.e. } z \in \mathbf{T} .
$$

If $d_{f}=0$, then the inequalities in (2.19) are obvious. Therefore we can assume that $d_{f}>0$, i.e., $\lambda>0$. Setting $G_{a}:=(\partial F)^{2}+a(\partial \bar{F})^{2}$ for $a \in \mathbf{C}$, we see that

$$
\begin{aligned}
\left|G_{a}(z)\right| & =\left|(\partial F(z))^{2}+a(\partial \bar{F}(z))^{2}\right| \geq|\partial F(z)|^{2}-|a||\bar{\partial} F(z)|^{2} \\
& =(1-|a|)|\partial F(z)|^{2}+|a| J[F](z) \geq(1-|a|)|\partial F(z)|^{2}, \quad a, z \in \mathbf{D} .
\end{aligned}
$$

By Kalaj's theorem,

$$
|\partial F(z)|^{2} \geq \frac{R_{1}^{2}}{32}, \quad z \in \mathbf{D}
$$

cf. [7, Thm. 2.5] and also [16, Thm. 2.2 and Cor. 3.1] for a stronger inequality. Combining this with $(2.22)$ we see that $1 / G_{a}$ is a bounded holomorphic function in $\mathbf{D}$ for each $a \in \mathbf{D}$. Applying now [2, Thm. 1.3] we see that for a given $a \in \mathbf{D} \backslash\{0\}$ there exists a function $g_{a}: \mathbf{T} \rightarrow \mathbf{C}$ such that

$$
g_{a}(z)=\lim _{r \rightarrow 1^{-}} \frac{1}{G_{a}(r z)} \quad \text { for a.e. } z \in \mathbf{T} .
$$

Hence, by (2.21) and by (2.22) it follows that for a.e. $z \in \mathbf{T}$,

$$
\left|g_{a}(z)\right|=\lim _{r \rightarrow 1^{-}} \frac{1}{\left|G_{a}(r z)\right|} \leq \lim _{r \rightarrow 1^{-}} \frac{1}{|a| J[F](r z)} \leq \frac{1}{\lambda|a|} .
$$

Therefore, for every $z \in \mathbf{D}$,

$$
\frac{1}{\left|G_{a}(z)\right|}=\left|\mathrm{P}\left[g_{a}\right](z)\right| \leq \mathrm{P}\left[\left|g_{a}\right|\right](z) \leq \mathrm{P}\left[\frac{1}{\lambda|a|}\right](z)=\frac{1}{\lambda|a|},
$$

and consequently,

$$
\left|G_{a}(z)\right| \geq \lambda|a|, \quad a, z \in \mathbf{D} .
$$

Given now $z \in \mathbf{D}$ we can choose $\alpha, \beta \in \mathbf{R}$ such that $(\partial F(z))^{2}=\mathrm{e}^{\mathrm{i} \alpha}|\partial F(z)|^{2}$ and $(\partial \bar{F}(z))^{2}=\mathrm{e}^{\mathrm{i} \beta}|\bar{\partial} F(z)|^{2}$. Setting $a:=-\mathrm{e}^{\mathrm{i}(\alpha-\beta)}$ we conclude from (2.23) that

$$
|\mathrm{J}[F](z)|=\left|\mathrm{e}^{\mathrm{i} \alpha} \mathrm{J}[F](z)\right|=\left|G_{a}(z)\right|=\lim _{r \rightarrow 1^{-}}\left|G_{r a}(z)\right| \geq \lim _{r \rightarrow 1^{-}} \lambda|r a|=\lambda,
$$

which yields the first inequality in (2.19). The second one follows directly from the inequality $\lambda \geq d_{f} R_{1} / 2$, which completes the proof.

Remark 2.7. Let $f$ be a sense-preserving homeomorphism of $\mathbf{T}$ onto the boundary of a bounded convex domain $\Omega$ in $\mathbf{C}$ such that $\mathrm{P}[f](0)=0$, and let $R>0$ satisfy the inclusion $\mathbf{D}(0, R) \subset \mathrm{P}[f](\mathbf{D})$. Then Theorem 2.5 and Corollary 2.6 imply in particular the following Kalaj's inequalities:

$$
\lim _{r \rightarrow 1^{-}} \mathrm{J}[\mathrm{P}[f]](r z) \geq \frac{d_{f} R}{2} \quad \text { for a.e. } z \in \mathbf{T}
$$


Quasiconformal and Lipschitz harmonic mappings of the unit disk onto bounded convex domains 821 as well as

$$
\mathrm{J}[\mathrm{P}[f]](z) \geq \frac{d_{f} R}{2}, \quad z \in \mathbf{D},
$$

respectively. In 2004 Kalaj proved them under the additional assumption that the function $f$ is absolutely continuous; cf. [8, Thm. 2.8 and Cor. 2.9]. Therefore, Theorem 2.5 and Corollary 2.6 considerably extend Kalaj's results.

\section{The Lipschitz property for harmonic mappings}

Given a nonempty set $\Omega \subset \mathbf{C}$ and a function $F: \Omega \rightarrow \mathbf{C}$ we denote by $\mathrm{L}(F)$ the Lipschitz constant of $F$, i.e.,

$$
\mathrm{L}(F):=\sup \left\{\left|\frac{F(z)-F(w)}{z-w}\right|: z, w \in \Omega, z \neq w\right\} .
$$

Note that $F$ is a Lipschitz function iff $\mathrm{L}(F)<+\infty$. If the last condition holds, then $F$ is a $L$-Lipschitz function for every $L \geq \mathrm{L}(F)$, i.e.,

$$
|F(w)-F(z)| \leq L|w-z|, \quad w, z \in \Omega .
$$

For any Lebesgue measurable function $f: \mathbf{T} \rightarrow \mathbf{C}$ we set

$$
\|f\|_{\infty}:=\operatorname{ess~sup}_{z \in \mathbf{T}}|f(z)| .
$$

Let $\mathrm{L}^{\infty}(\mathbf{T})$ denote the class of all such functions $f$ with $\|f\|_{\infty}<+\infty$.

Lemma 3.1. If $f: \mathbf{T} \rightarrow \mathbf{C}$ is a function of bounded variation, then $F:=\mathrm{P}[f]$ satisfies the following inequalities

$$
\frac{1}{2}\left\||\mathrm{~A}[\mathrm{~d} f]|^{2}+|\dot{f}|^{2}\right\|_{\infty} \leq \mathrm{L}(F)^{2} \leq\left\||\mathrm{A}[\mathrm{d} f]|^{2}+|\dot{f}|^{2}\right\|_{\infty} .
$$

Proof. Given a function of bounded variation $f: \mathbf{T} \rightarrow \mathbf{C}$ we know by Corollary 1.3 that all the limits in (1.20), (1.21) and (1.22) exist for a.e. $z \in \mathbf{T}$. Choosing arbitrarily such a $z$ we deduce from (1.21) and (1.22) that

$$
2 \lim _{r \rightarrow 1^{-}}\left(|\partial \mathrm{P}[f](r z)|^{2}+|\bar{\partial} \mathrm{P}[f](r z)|^{2}\right)=|\mathrm{A}[\mathrm{d} f](z)|^{2}+\left|f^{\prime}(z)\right|^{2} \quad \text { for a.e. } z \in \mathbf{T} \text {. }
$$

Since

$$
F(w)-F(u)=\partial F(u)(w-u)+\bar{\partial} F(u) \overline{(w-u)}+\mathrm{o}(w-u), \quad w, u \in \mathbf{D},
$$

we conclude from (3.1) that

$$
\sup _{u \in \mathbf{D}}(|\partial F(u)|+|\bar{\partial} F(u)|)=\mathrm{L}(F) .
$$

Assume first that $F$ is a Lipschitz function, i.e., $\mathrm{L}(F)<+\infty$. Then the equality (3.6) leads to

$$
|\partial F(u)|^{2}+|\bar{\partial} F(u)|^{2} \leq \mathrm{L}(F)^{2}, \quad u \in \mathbf{D} .
$$

Combining this with (3.4) we obtain the first inequality in (3.3). On the other hand from (3.7) it follows that both the functions $\partial F$ and $\bar{\partial} F$ are holomorphic and bounded 
in D. Moreover, from (1.21) and (1.22) we see that there exist bounded functions $f_{1}, f_{2}: \mathbf{T} \rightarrow \mathbf{C}$ such that for a.e. $z \in \mathbf{T}$,

$$
\begin{aligned}
\lim _{r \rightarrow 1^{-}} \partial \mathrm{P}[f](r z) & =f_{1}(z)=\frac{\bar{z}}{2}\left(\mathrm{~A}[\mathrm{~d} f](z)+z f^{\prime}(z)\right), \\
\lim _{r \rightarrow 1^{-}} \bar{\partial} \mathrm{P}[f](r z) & =f_{2}(z)=\frac{z}{2}\left(\mathrm{~A}[\mathrm{~d} f](z)-z f^{\prime}(z)\right) .
\end{aligned}
$$

Thus, applying the Schwarz integral inequality, we obtain

$$
\begin{aligned}
|\partial F(u)|^{2}+|\bar{\partial} F(u)|^{2} & =\left|\mathrm{P}\left[f_{1}\right](u)\right|^{2}+\left|\mathrm{P}\left[f_{2}\right](u)\right|^{2} \leq \mathrm{P}\left[\left|f_{1}\right|^{2}\right](u)+\mathrm{P}\left[\left|f_{2}\right|^{2}\right](u) \\
& =\mathrm{P}\left[\left|f_{1}\right|^{2}+\left|f_{2}\right|^{2}\right](u)=\frac{1}{2} \mathrm{P}\left[|\mathrm{A}[\mathrm{d} f]|^{2}+\left|f^{\prime}\right|^{2}\right](u), \quad u \in \mathbf{D} .
\end{aligned}
$$

Combining this with (3.6) we get

$$
\begin{aligned}
\mathrm{L}(F)^{2} & \leq 2 \sup _{u \in \mathbf{D}}\left(|\partial F(u)|^{2}+|\bar{\partial} F(u)|^{2}\right) \leq \sup _{u \in \mathbf{D}} \mathrm{P}\left[|\mathrm{A}[\mathrm{d} f]|^{2}+\left|f^{\prime}\right|^{2}\right](u) \\
& \leq\left\||\mathrm{A}[\mathrm{d} f]|^{2}+\left|f^{\prime}\right|^{2}\right\|_{\infty},
\end{aligned}
$$

and so the second inequality in (3.3) holds.

It remains to consider the case where $F$ is not a Lipschitz function, i.e., $\mathrm{L}(F)=$ $+\infty$. Then the first inequality in (3.3) is obvious. In order to show the second inequality in (3.3) suppose that $\left\||\mathrm{A}[\mathrm{d} f]|^{2}+\left|f^{\prime}\right|^{2}\right\|_{\infty}<+\infty$. Then $\dot{f} \in \mathrm{L}^{\infty}(\mathbf{T})$ and $\mathrm{A}[\mathrm{d} f] \in \mathrm{L}^{\infty}(\mathbf{T})$. Hence and by (1.21) and (1.22) we see that both the functions $\partial \mathrm{P}[f]$ and $\bar{\partial} \mathrm{P}[f]$ have essentially bounded radial limiting valued functions. Furthermore, by Corollary 1.3, $\partial \mathrm{P}[f], \bar{\partial} \mathrm{P}[f] \in \mathrm{H}^{1 / 2}(\mathbf{D})$. Therefore both the functions $\partial \mathrm{P}[f]$ and $\bar{\partial} \mathrm{P}[f]$ are uniquely determined by the Poisson integral operator from their radial

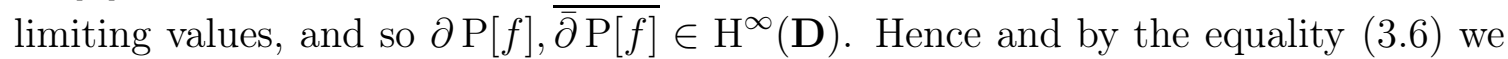
deduce that $\mathrm{L}(F)<+\infty$, which contradicts our assumption. Therefore $\||\mathrm{A}[\mathrm{d} f]|^{2}+$ $\left|f^{\prime}\right|^{2} \|_{\infty}=+\infty$, and so the second inequality in (3.3) holds, which completes the proof.

The following theorem generalizes and improves the equivalence (i) $\Longleftrightarrow$ (iv) in [14, Thm. 2.2].

Theorem 3.2. Let $F: \mathbf{D} \rightarrow \mathbf{C}$ be a harmonic mapping. If $F$ is a Lipschitz mapping, then $F$ has the continuous extension to the closure $\mathrm{cl}(\mathbf{D})$ and its boundary limiting valued function $f$ is absolutely continuous and satisfies the following condition

$$
\|\mathrm{A}[\dot{f}]\|_{\infty} \leq \sqrt{2} \mathrm{~L}(F), \quad\|\dot{f}\|_{\infty} \leq \mathrm{L}(F) \quad \text { and } \quad \mathrm{L}(f) \leq \mathrm{L}(F) .
$$

Conversely, if $F$ has the continuous extension to $\mathrm{cl}(\mathbf{D})$ and its boundary limiting valued function $f$ is absolutely continuous, $\mathrm{A}[\dot{f}] \in \mathrm{L}^{\infty}(\mathbf{T})$ and $\dot{f} \in \mathrm{L}^{\infty}(\mathbf{T})$, then $F$ is a Lipschitz mapping with

$$
\mathrm{L}(F) \leq \sqrt{\|\mathrm{A}[\dot{f}]\|_{\infty}^{2}+\|\dot{f}\|_{\infty}^{2}} .
$$

Proof. Fix a harmonic mapping $F: \mathbf{D} \rightarrow \mathbf{C}$. Assume that $F$ is a Lipschitz mapping. Then $F$ is uniformly continuous, and so it has the unique continuous extension $F^{*}$ to $\operatorname{cl}(\mathbf{D})$. A passage to the limit implies that $f:=F_{\mid \mathbf{T}}^{*}$ is a Lipschitz function with $\mathrm{L}(f) \leq \mathrm{L}\left(F^{*}\right)=\mathrm{L}(F)$. Therefore the third and-consequently-the second inequalities in (3.8) hold. In particular, $f$ is an absolutely continuous function, 
and so the equality (1.18) holds. Since $F=\mathrm{P}[f]$, we infer from Lemma 3.1 the first inequality in (3.8).

Conversely, assume that $F$ has the continuous extension $F^{*}$ to $\operatorname{cl}(\mathbf{D})$ and $f$ is absolutely continuous, $\mathrm{A}[\dot{f}] \in \mathrm{L}^{\infty}(\mathbf{T})$ and $\dot{f} \in \mathrm{L}^{\infty}(\mathbf{T})$. Since $F=\mathrm{P}[f]$, we conclude from Lemma 3.1 that $F$ is a Lipschitz mapping with $\mathrm{L}(F)$ satisfying the inequality (3.9), which completes the proof.

Given a simply connected domain $\Omega$ in $\mathbf{C}$ and a harmonic mapping $F: \Omega \rightarrow \mathbf{C}$ there exist holomorphic functions $H$ and $G$ in $\Omega$ such that $F=H+\bar{G}$. The functions $H$ and $G$ are determined up to the constant. Any such function $H$ (resp. $G$ ) is said to be a holomorphic part of $F$ (resp. an anti-holomorphic part of $F$ ). We recall that a domain $\Omega$ in $\mathbf{C}$ is said to be linearly connected if there exists a constant $M \geq 1$ such that any points $z, w \in \Omega$ can be connected by a path $\gamma$ with length $|\gamma|_{1} \leq M|z-w|$. Write $\mathbf{Z}_{p, q}:=\{n \in \mathbf{Z}: p \leq n \leq q\}$ for any $p, q \in \mathbf{R}$.

Lemma 3.3. If $\Omega$ is a linearly connected domain and $F: \Omega \rightarrow \mathbf{C}$ is a bi-Lipschitz mapping, then $F(\Omega)$ is a linearly connected domain.

Proof. Given $a, b \in \mathbf{R}, a<b$, suppose that $\gamma:[a ; b] \rightarrow \Omega$ is a rectifiable path. Then for arbitrarily fixed $\varepsilon>0$ there exist $n \in \mathbf{N}$ and an increasing sequence $\mathbf{Z}_{0, n} \ni k \mapsto t_{k} \in[a ; b]$ such that $t_{0}=a, t_{n}=b$ and

$$
\sum_{k=1}^{n}\left|F \circ \gamma\left(t_{k}\right)-F \circ \gamma\left(t_{k-1}\right)\right|>|F \circ \gamma|_{1}-\varepsilon .
$$

Since $F$ is a Lipschitz mapping, we see that

$$
\begin{aligned}
|F \circ \gamma|_{1} & <\sum_{k=1}^{n}\left|F \circ \gamma\left(t_{k}\right)-F \circ \gamma\left(t_{k-1}\right)\right|+\varepsilon \\
& \leq \mathrm{L}(F) \sum_{k=1}^{n}\left|\gamma\left(t_{k}\right)-\gamma\left(t_{k-1}\right)\right|+\varepsilon \leq \mathrm{L}(F)|\gamma|_{1}+\varepsilon .
\end{aligned}
$$

Therefore $|F \circ \gamma|_{1} \leq \mathrm{L}(F)|\gamma|_{1}$, because $\varepsilon>0$ can be arbitrarily small. Since $F^{-1}$ is also a Lipschitz mapping, we may apply the above reasoning, with replacing $F$ by $F^{-1}$ and $\gamma$ by $F \circ \gamma$, to obtain $|\gamma|_{1}=\left|F^{-1} \circ(F \circ \gamma)\right|_{1} \leq \mathrm{L}\left(F^{-1}\right)|F \circ \gamma|_{1}$. Thus

$$
\frac{1}{\mathrm{~L}\left(F^{-1}\right)}|\gamma|_{1} \leq|F \circ \gamma|_{1} \leq \mathrm{L}(F)|\gamma|_{1} \text {. }
$$

Assume now that $\Omega$ is a linearly connected domain and fix $z, w \in F(\Omega)$. Then there exists a path $\gamma:[a ; b] \rightarrow \Omega$ such that $\gamma(a)=F^{-1}(z)$ and $\gamma(b)=F^{-1}(w)$, such that

$$
|\gamma|_{1} \leq M\left|F^{-1}(z)-F^{-1}(w)\right| \leq M \mathrm{~L}\left(F^{-1}\right)|z-w|,
$$

where $M \geq 1$ is a constant which depends on $\Omega$ only. Combining this with the second inequality in (3.10) we have

$$
|F \circ \gamma|_{1} \leq \mathrm{L}(F)|\gamma|_{1} \leq M \mathrm{~L}(F) \mathrm{L}\left(F^{-1}\right)|z-w| .
$$

Since $F \circ \gamma:[a ; b] \rightarrow F(\Omega)$ is a path connecting the points $z$ and $w$, we conclude from (3.11) that $F(\Omega)$ is a linearly connected domain, because the constant $M \mathrm{~L}(F) \mathrm{L}\left(F^{-1}\right)$ does not depend on the choice of the points $z, w \in F(\Omega)$. This is the desired conclusion. 
Theorem 3.4. Let $F: \mathbf{D} \rightarrow \mathbf{C}$ be a sense-preserving injective harmonic mapping such that $F(\mathbf{D})$ is a bounded convex domain in $\mathbf{C}$. Then the following conditions are equivalent to each other:

(i) $F$ is a quasiconformal and Lipschitz mapping;

(ii) $F$ is a quasiconformal mapping and its boundary limiting valued function $f$ is a Lipschitz mapping;

(iii) $F$ is a quasiconformal mapping and a holomorphic part $H$ of $F$ is a biLipschitz mapping;

(iv) $F$ is a bi-Lipschitz mapping;

(v) $F$ has a continuous extension to the closure $\mathrm{cl}(\mathbf{D})$ and its boundary limiting valued function $f$ is absolutely continuous and satisfies the following condition

$$
0<d_{f}, \quad\|\dot{f}\|_{\infty}<+\infty \text { and }\|\mathrm{A}[\dot{f}]\|_{\infty}<+\infty
$$

where $d_{f}$ is defined by $(2.20)$.

Proof. Given a sense-preserving injective harmonic mapping $F=H+\bar{G}: \mathbf{D} \rightarrow \mathbf{C}$ suppose that $\Omega:=F(\mathbf{D})$ is a bounded convex domain in $\mathbf{C}$, where $H$ and $G$ are a holomorphic part of $F$ and an anti-holomorphic part of $F$, respectively. We may assume additionally that $H(0)=0=G(0)$. Since $\Omega$ is convex, the mapping $H$ is injective; cf. [1, Cor. 5.8] or [17, Cor. 2.2].

First suppose that the condition (i) holds. From Theorem 3.2 it follows that the boundary limiting valued function $f$ of $F$ is a Lipschitz mapping, and so the implication (i) $\Longrightarrow$ (ii) holds.

Next suppose that the condition (ii) holds. From [15, Lem. 2.3] it follows that

$$
|\partial F(z)| \leq \frac{K+1}{2} \mathrm{~L}(f), \quad z \in \mathbf{D},
$$

where $K$ is the maximal dilatation of $F$. Thus for all $z, w \in \mathbf{D}$ we see, setting $[0 ; 1] \ni t \mapsto \gamma(t):=(1-t) z+t w \in \mathbf{D}$, that

$$
\begin{aligned}
|H(w)-H(z)| & =\int_{0}^{1}\left|\frac{\mathrm{d}}{\mathrm{d} t}(H \circ \gamma)(t)\right| \mathrm{d} t \leq \int_{0}^{1}\left|H^{\prime}(\gamma(t))\right|\left|\gamma^{\prime}(t)\right| \mathrm{d} t \\
& \leq \int_{0}^{1}|\partial F(\gamma(t))| \mathrm{d} t \cdot|w-z| \leq \frac{K+1}{2} \mathrm{~L}(f)|w-z| .
\end{aligned}
$$

Therefore $H$ is a Lipschitz mapping with $\mathrm{L}(H) \leq \frac{K+1}{2} \mathrm{~L}(f)$.

From [17, Thm. 3.8] it follows that $H \circ F^{-1}$ is a bi-Lipschitz mapping. By the equality $H=\left(H \circ F^{-1}\right) \circ F$ we obtain

$$
H(\mathbf{D})=H \circ F^{-1}(F(\mathbf{D}))=H \circ F^{-1}(\Omega) .
$$

Since $\Omega$ is a convex domain, $\Omega$ is linearly connected with respect to a constant $M:=1$. Therefore from Lemma 3.3 it follows that $H(\mathbf{D})$ is a linearly connected domain with a new constant $M \geq 1$. As $0=F(0) \in \Omega$, there exists $R>0$ such that $\mathbf{D}(0, R) \subset \Omega$, where $\mathbf{D}(a, r):=\{z \in \mathbf{C}:|z-a|<r\}$ for $a \in \mathbf{C}$ and $r>0$. Applying now [16, Cor. 3.1] we see that

$$
\left|H^{\prime}(z)\right|=|\partial F(z)| \geq \frac{R}{4}, \quad z \in \mathbf{D} .
$$

Fix distinct points $z, w \in \mathbf{D}$. Since the domain $H(\mathbf{D})$ is linearly connected, there exists a path $\gamma:[0 ; 1] \rightarrow H(\mathbf{D})$ connecting the points $\gamma(0)=H(z)$ and $\gamma(1)=H(w)$ 
such that $|\gamma|_{1} \leq M|H(w)-H(z)|$. Since $\gamma$ is an uniformly continuous mapping, the image $\gamma([0 ; 1])$ is a compact subset of the domain $H(\Omega)$, and consequently there exist $r>0$ and $\delta>0$ such that

$$
\gamma([t-\delta ; t+\delta] \cap[0 ; 1]) \subset \mathbf{D}(\gamma(t), r) \subset H(\Omega), \quad t \in[0 ; 1] .
$$

Let us consider $n \in \mathbf{N}$ and an increasing sequence $\mathbf{Z}_{0, n} \ni k \mapsto t_{k} \in[a ; b]$ such that $t_{0}=0, t_{n}=1$ and $t_{k}-t_{k-1}<\delta$ for $k \in \mathbf{Z}_{1, n}$. Hence and by (3.15), the mappings

$$
[0 ; 1] \ni s \mapsto \gamma_{k}(s):=H^{-1}\left(s \gamma\left(t_{k}\right)+(1-s) \gamma\left(t_{k-1}\right)\right) \in \mathbf{D}, \quad k \in \mathbf{Z}_{1, n},
$$

are well defined. Moreover, each mapping $\gamma_{k}$ is a regular path in $\mathbf{D}$ connecting the points $H^{-1}\left(\gamma\left(t_{k-1}\right)\right)$ and $H^{-1}\left(\gamma\left(t_{k}\right)\right)$. By (3.14) we have

$$
\begin{aligned}
|\gamma|_{1} & \geq \sum_{k=1}^{n}\left|\gamma\left(t_{k}\right)-\gamma\left(t_{k-1}\right)\right|=\sum_{k=1}^{n}\left|H\left(H^{-1}\left(\gamma\left(t_{k}\right)\right)\right)-H\left(H^{-1}\left(\gamma\left(t_{k-1}\right)\right)\right)\right| \\
& =\sum_{k=1}^{n} \int_{0}^{1}\left|\frac{\mathrm{d}}{\mathrm{d} t}\left(H \circ \gamma_{k}\right)(t)\right| \mathrm{d} t=\sum_{k=1}^{n} \int_{0}^{1}\left|H^{\prime}\left(\gamma_{k}(t)\right)\right|\left|\gamma_{k}^{\prime}(t)\right| \mathrm{d} t \\
& \geq \frac{R}{4} \sum_{k=1}^{n} \int_{0}^{1}\left|\gamma_{k}^{\prime}(t)\right| \mathrm{d} t \geq \frac{R}{4}\left|\sum_{k=1}^{n} \int_{0}^{1} \gamma_{k}^{\prime}(t) \mathrm{d} t\right|=\frac{R}{4}\left|\sum_{k=1}^{n}\left(\gamma_{k}(1)-\gamma_{k}(0)\right)\right| \\
& =\frac{R}{4}\left|\sum_{k=1}^{n}\left(H^{-1}\left(\gamma\left(t_{k}\right)\right)-H^{-1}\left(\gamma\left(t_{k-1}\right)\right)\right)\right|=\frac{R}{4}\left|H^{-1}\left(\gamma\left(t_{n}\right)\right)-H^{-1}\left(\gamma\left(t_{0}\right)\right)\right| \\
& =\frac{R}{4}\left|H^{-1}(\gamma(1))-H^{-1}(\gamma(0))\right|=\frac{R}{4}|w-z| .
\end{aligned}
$$

Combining this with the inequality $|\gamma|_{1} \leq M|H(w)-H(z)|$ we conclude that

$$
|H(w)-H(z)| \geq \frac{1}{M}|\gamma|_{1} \geq \frac{R}{4 M}|w-z|, \quad w, z \in \mathbf{D} .
$$

Thus $H$ is a co-Lipschitz mapping. Since $H$ is also a Lipschitz mapping, we conclude that $H$ is a bi-Lipschitz one. This proves the implication (ii) $\Longrightarrow$ (iii).

Suppose now that the condition (iii) holds. Then [17, Thm. 3.8] shows that the composite mapping $F \circ H^{-1}$ is bi-Lipschitz. Hence $F$ is a bi-Lipschitz mapping since the mapping $H$ is so and $F=\left(F \circ H^{-1}\right) \circ H$, which yields the implication (iii) $\Longrightarrow$ (iv).

Supposing that the condition (iv) holds, we see that $\mathrm{L}(F)<+\infty$ and $\mathrm{L}\left(F^{-1}\right)<$ $+\infty$. Then by Theorem 3.2, $F$ has the continuous extension to the closure cl(D) and its boundary limiting valued function $f$ is absolutely continuous and satisfies the condition (3.8). Setting $L:=\mathrm{L}\left(F^{-1}\right)$ we see that

$$
\frac{1}{L}|w-z| \leq|F(w)-F(z)|, \quad w, z \in \mathbf{D} \text {. }
$$

Hence for all $t, s \in \mathbf{R}$ and $r \in[0 ; 1)$,

$$
\left|\mathrm{e}^{\mathrm{i} t}-\mathrm{e}^{\mathrm{i} \theta}\right|=\lim _{r \rightarrow 1^{-}}\left|r \mathrm{e}^{\mathrm{i} t}-r \mathrm{e}^{\mathrm{i} \theta}\right| \leq L \lim _{r \rightarrow 1^{-}}\left|F\left(r \mathrm{e}^{\mathrm{i} t}\right)-F\left(r \mathrm{e}^{\mathrm{i} \theta}\right)\right|=L\left|f\left(\mathrm{e}^{\mathrm{i} t}\right)-f\left(\mathrm{e}^{\mathrm{i} \theta}\right)\right| .
$$

Given $\theta \in \mathbf{R}$ assume that there exists the derivative $\dot{f}(z)$ at the point $z:=\mathrm{e}^{\mathrm{i} \theta}$. Then

$$
|\dot{f}(z)|=\left|\lim _{t \rightarrow \theta} \frac{f\left(\mathrm{e}^{\mathrm{i} t}\right)-f\left(\mathrm{e}^{\mathrm{i} \theta}\right)}{t-\theta}\right| \geq \frac{1}{L}\left|\lim _{t \rightarrow \theta} \frac{\mathrm{e}^{\mathrm{i} t}-\mathrm{e}^{\mathrm{i} \theta}}{t-\theta}\right|=\frac{1}{L}\left|\mathrm{i} \mathrm{i}^{\mathrm{i} \theta}\right|=\frac{1}{L} .
$$


Thus $|\dot{f}(z)| \geq 1 / L$ for a.e. $z \in \mathbf{T}$, and so $d_{f} \geq 1 / L$. Combining this with (3.8) we obtain (3.12), which completes the proof of the implication (iv) $\Longrightarrow(v)$.

Suppose finally that the condition (v) holds. Then $f$ is an absolutely continuous function and $F=\mathrm{P}[f]$. Since $F$ is a sense-preserving injective harmonic mapping, we conclude from Levy's theorem that the Jacobian $\mathrm{J}[F]$ is positive in $\mathbf{D}$; cf. [10]. Therefore

$$
|\partial F(z)|^{2}-|\bar{\partial} F(z)|^{2}=\mathrm{J}[F](z)>0, \quad z \in \mathbf{D}
$$

and so the second dilatation

$$
\mathbf{D} \ni z \mapsto \omega(z):=\frac{\overline{\overline{\partial F(z)}}}{\partial F(z)}=\frac{G^{\prime}(z)}{H^{\prime}(z)}
$$

of $F$ is well defined as well as

$$
|\omega(z)|<1, \quad z \in \mathbf{D} .
$$

Applying now [2, Thm. 1.3] we see that there exists a function $\omega^{*}: \mathbf{T} \rightarrow \mathbf{C}$ such that

$$
\omega^{*}(z)=\lim _{r \rightarrow 1^{-}} \omega(r z) \quad \text { for a.e. } z \in \mathbf{T} .
$$

From (1.3) and Corollary 1.3 it follows that for a.e. $z \in \mathbf{T}$,

$$
2 \lim _{r \rightarrow 1^{-}} \partial F(r z)=\bar{z}(\mathrm{~A}[\dot{f}](z)-\mathrm{i} \dot{f}(z)),
$$

and consequently

$$
4\left|\lim _{r \rightarrow 1^{-}} \partial F(r z)\right|^{2}=|\mathrm{A}[\dot{f}](z)-\mathrm{i} \dot{f}(z)|^{2} \leq 2|\mathrm{~A}[\dot{f}](z)|^{2}+2|\dot{f}(z)|^{2} .
$$

Combining this with (3.12) we obtain

$$
\left|\lim _{r \rightarrow 1^{-}} \partial F(r z)\right|^{2} \leq M_{f}:=\frac{1}{2}\|\mathrm{~A}[\dot{f}]\|_{\infty}^{2}+\frac{1}{2}\|\dot{f}\|_{\infty}^{2}<+\infty \quad \text { for a.e. } z \in \mathbf{T} .
$$

As $0=F(0) \in \Omega$ and $\Omega$ is a bounded domain in $\mathbf{C}$, there exist $R_{1}, R_{2}>0$ satisfying the condition (2.18). Since additionally $\Omega$ is a convex domain, we conclude from Remark 2.2 that each point $f(z), z \in \mathbf{T}$, is linearly accessible from outside of $\Omega$. Corollary 1.3 and Theorem 2.5 now show that the inequalities (2.17) hold for a.e. $z \in \mathbf{T}$. Hence and by (3.12),

$$
\lim _{r \rightarrow 1^{-}} \mathrm{J}[F](r z) \geq\left|f^{\prime}(z)\right| \frac{R_{1}}{2} \geq \frac{R_{1} d_{f}}{2}>0 \quad \text { for a.e. } z \in \mathbf{T} .
$$

Combining this with (3.16), (3.17), (3.19) and (3.20) we see that for a.e. $z \in \mathbf{T}$,

$$
M_{f}\left(1-\left|\omega^{*}(z)\right|^{2}\right) \geq \lim _{r \rightarrow 1^{-}}|\partial F(r z)|^{2}\left(1-|\omega(r z)|^{2}\right)=\lim _{r \rightarrow 1^{-}} J[F](r z) \geq \frac{R_{1} d_{f}}{2} .
$$

Hence

$$
\left\|\omega^{*}\right\|_{\infty}^{2} \leq 1-\frac{R_{1} d_{f}}{2 M_{f}}<1
$$

This implies, by (3.17) and (3.18), that

$$
\left|\frac{\bar{\partial} F(z)}{\partial F(z)}\right|=|\omega(z)|=\left|\mathrm{P}\left[\omega^{*}\right](z)\right| \leq\left\|\omega^{*}\right\|_{\infty} \leq \sqrt{1-\frac{R_{1} d_{f}}{2 M_{f}}}<1, \quad z \in \mathbf{D} .
$$

Therefore $F$ is a quasiconformal mapping. By Theorem 3.2, $F$ is also a Lipschitz mapping. This leads to the implication $(\mathrm{v}) \Longrightarrow(\mathrm{i})$, which completes the proof. 
Remark 3.5. Suppose that $\Omega \subset \mathbf{C}$ is a bounded convex domain and that $f$ is a sense-preserving homeomorphism of $\mathbf{T}$ onto the boundary of $\Omega$. Then by RadóKneser-Choquet Theorem $F:=\mathrm{P}[f]$ is a sense-preserving injective harmonic mapping of $\mathbf{D}$ onto $\Omega$. Under these assumptions $\mathrm{Zhu}$ has shown recently the equivalence of the conditions (iv) and (v) in our Theorem 3.4; cf. [21, Thm. 3]. Suppose additionally that the function $f$ is absolutely continuous on $\mathbf{T}$. Then from Kalaj's theorem [8, Thm. 3.2] it follows that the conditions (i) and (ii) in our Theorem 3.4 are equivalent. In this sense, Theorem 3.4 is a generalization of both results by Zhu and Kalaj.

\section{Applications}

We recall that a function $f: \mathbf{T} \rightarrow \mathbf{C}$ is said to be Dini-smooth if $f$ is differentiable on $\mathbf{T}$ and the derivative $\dot{f}$ is not vanishing and Dini-continuous on $\mathbf{T}$, i.e., its modulus of continuity

$$
\omega(\delta):=\sup \left\{\left|\dot{f}\left(\mathrm{e}^{\mathrm{i} t}\right)-\dot{f}\left(\mathrm{e}^{\mathrm{i} s}\right)\right|: t, s \in \mathbf{R},|t-s| \leq \delta\right\}, \quad \delta \in[0 ; \pi],
$$

satisfies the following condition

$$
D_{f}:=\int_{0}^{\pi} \frac{\omega(t)}{t} \mathrm{~d} t<+\infty .
$$

Corollary 4.1. Let $f: \mathbf{T} \rightarrow \mathbf{C}$ be a Dini-smooth and injective function. If $f(\mathbf{T})$ is the boundary curve of a convex domain $\Omega$ in $\mathbf{C}$, then $d_{f}>0,\|\dot{f}\|_{\infty}<+\infty$ and $F:=\mathrm{P}[f]$ is a bi-Lipschitz mapping of $\mathbf{D}$ onto $\Omega$ with

$$
\mathrm{L}(F) \leq \sqrt{\frac{4}{\pi^{2}} D_{f}^{2}+\|\dot{f}\|_{\infty}^{2}}
$$

and

$$
\frac{1}{\mathrm{~L}\left(F^{-1}\right)} \geq \frac{d_{f}}{\mathrm{~L}(F)} \cdot \frac{R_{1}+R_{2}}{\pi} \tan \left(\frac{\pi}{2} \frac{R_{1}}{R_{1}+R_{2}}\right) \geq \frac{d_{f} R_{1}}{2 \mathrm{~L}(F)}
$$

provided $R_{1}, R_{2}>0$ satisfy

$$
\mathbf{D}\left(F(0), R_{1}\right) \subset \Omega \subset \mathbf{D}\left(F(0), R_{2}\right) .
$$

In particular, if additionally $\mathrm{J}[F](0)>0$, then $F$ is $\mathrm{L}(F) \mathrm{L}\left(F^{-1}\right)$-quasiconformal.

Proof. Fix a function $f: \mathbf{T} \rightarrow \Gamma$ satisfying the assumption. Then $f$ is differentiable at every point of $\mathbf{T}$ and the derivative $\dot{f}$ is not vanishing and Dini-continuous on $\mathbf{T}$. Therefore $\dot{f}$ is continuous on $\mathbf{T}$, and so

$$
d_{f}=\min _{z \in \mathbf{T}}|\dot{f}(z)|>0 \quad \text { and } \quad\|\dot{f}\|_{\infty}=\max _{z \in \mathbf{T}}|\dot{f}(z)|<+\infty
$$

because $\mathbf{T}$ is a compact set. From (4.1) it follows that for every $\theta \in \mathbf{R}$ and $\varepsilon \in(0 ; \pi)$,

$$
\begin{aligned}
\left|\int_{\varepsilon<|t-\theta| \leq \pi} \cot \frac{\theta-t}{2} \dot{f}\left(\mathrm{e}^{\mathrm{i} t}\right) \mathrm{d} t\right| & =\left|\int_{\varepsilon<|t-\theta| \leq \pi} \cot \frac{\theta-t}{2}\left(\dot{f}\left(\mathrm{e}^{\mathrm{i} t}\right)-\dot{f}\left(\mathrm{e}^{\mathrm{i} \theta}\right)\right) \mathrm{d} t\right| \\
& \leq \int_{\varepsilon<|t-\theta| \leq \pi}\left|\cot \frac{\theta-t}{2}\right|\left|\dot{f}\left(\mathrm{e}^{\mathrm{i} t}\right)-\dot{f}\left(\mathrm{e}^{\mathrm{i} \theta}\right)\right| \mathrm{d} t \\
& \leq \int_{\varepsilon<|t-\theta| \leq \pi} \omega(|\theta-t|)\left|\cot \frac{\theta-t}{2}\right| \mathrm{d} t
\end{aligned}
$$




$$
=2 \int_{\varepsilon}^{\pi} \frac{\omega(t)}{t} \cdot t \cot \frac{t}{2} \mathrm{~d} t \leq 4 \int_{\varepsilon}^{\pi} \frac{\omega(t)}{t} \mathrm{~d} t \leq 4 D_{f}
$$

because $x \cot x \leq \tan x \cdot \cot x=1$ for $x \in(0 ; \pi / 2)$. Applying now [5, Chap. III, Lem. 1.2] we see that for a.e. $\mathrm{e}^{\mathrm{i} \theta} \in \mathbf{T}$,

$$
\left|\mathrm{A}[\dot{f}]\left(\mathrm{e}^{\mathrm{i} \theta}\right)\right|=\lim _{\varepsilon \rightarrow 0^{+}} \frac{1}{2 \pi}\left|\int_{\varepsilon<|t-\theta| \leq \pi} \cot \frac{\theta-t}{2} \dot{f}\left(\mathrm{e}^{\mathrm{i} t}\right) \mathrm{d} t\right| \leq \frac{2}{\pi} D_{f}
$$

Therefore,

$$
\|\mathrm{A}[\dot{f}]\|_{\infty} \leq \frac{2}{\pi} D_{f}
$$

Applying the classical Lagrange's mean value theorem to the real and imaginary parts of the function $\mathbf{R} \ni t \mapsto f\left(\mathrm{e}^{\mathrm{i} t}\right)$ we conclude from (4.5) that

$$
\left|f\left(\mathrm{e}^{\mathrm{i} t}\right)-f\left(\mathrm{e}^{\mathrm{i} s}\right)\right| \leq \sqrt{2}\|\dot{f}\|_{\infty}|t-s|, \quad t, s \in \mathbf{R},
$$

and consequently $f$ is absolutely continuous. In particular, $f$ is continuous, and hence the mapping $F$ has a continuous extension $F^{*}$ to the closed $\operatorname{disk} \operatorname{cl}(\mathbf{D})$ and $f=F_{\mid \mathbf{T}}^{*}$. Since $f$ is additionally injective on $\mathbf{T}, f$ is a homeomorphism of $\mathbf{T}$ onto $\Gamma:=f(\mathbf{T})$. Furthermore, $\Gamma$ is the boundary curve of the convex domain $\Omega$. Then the classical Radó-Kneser-Choquet theorem implies that $F$ is an injective harmonic mapping of $\mathbf{D}$ onto $\Omega$; cf. [3, Sect. 3.1]. Consequently, by the Lewy theorem $\mathrm{J}[F](z) \neq 0$ for $z \in \mathbf{D}$. Therefore $\mathrm{J}[F](z)>0$ for $z \in \mathbf{D}$ provided $\mathrm{J}[F](0)>0$. Assume now that $\mathrm{J}[F](0)>0$. Then Theorem 3.4 shows that the mapping $F$ is simultaneously biLipschitz and quasiconformal. Furthermore, from Theorem 3.2 and (4.6) we obtain the inequality (4.2). Since $\Omega$ is a convex domain we can follow the proof of $[15$, Thm. 3.3] to obtain the following estimation

$$
\begin{aligned}
|F(z)-F(w)| & \geq \inf _{u \in \mathbf{D}} \frac{|\partial F(u)|^{2}-|\bar{\partial} F(u)|^{2}}{|\partial F(u)|+|\bar{\partial} F(u)|}|z-w| \\
& \geq \frac{\inf _{u \in \mathbf{D}} \mathrm{J}[F](u)}{\sup _{u \in \mathbf{D}}(|\partial F(u)|+|\bar{\partial} F(u)|)}|z-w|, \quad z, w \in \mathbf{D} .
\end{aligned}
$$

If $F(0)=0$, then the condition (4.4) becomes (2.18). Applying now Corollary 2.6 and the equality (3.6) we infer the inequality (4.3). If $F(0) \neq 0$, then we can adopt this reasoning to the mapping $F-F(0)$ in place of the mapping $F$. Since the mapping $F$ is bi-Lipschitz, it is also $\mathrm{L}(F) \mathrm{L}\left(F^{-1}\right)$-quasiconformal.

In the case where $\mathrm{J}[F](0)<0$ we have $\mathrm{J}[F](z)<0$ for $z \in \mathbf{D}$. Then $\mathrm{J}[\bar{F}](z)>0$ for $z \in \mathbf{D}$. Since $\mathrm{P}[\bar{f}]=\bar{F}$, we may follow the above reasoning with $f$ replaced by $\bar{f}$. As a result we see that $\bar{F}$ is a bi-Lipschitz mapping. Then the mapping $F$ is also bi-Lipschitz and the inequalities (4.2) and (4.3) hold, which completes the proof.

Let us recall that a function $f: \mathbf{T} \rightarrow \mathbf{C}$ is said to be Hölder-smooth if $f$ is differentiable on $\mathbf{T}$ and the derivative $\dot{f}$ is not vanishing and Hölder-continuous on T, i.e., there exist $L \geq 0$ and $\alpha \in(0 ; 1]$ such that

$$
\left|\dot{f}\left(\mathrm{e}^{\mathrm{i} t}\right)-\dot{f}\left(\mathrm{e}^{\mathrm{i} s}\right)\right| \leq L|t-s|^{\alpha}, \quad t, s \in \mathbf{R} .
$$


Remark 4.2. It is clear that each Hölder continuous function is a Dini-continuous one. Therefore Corollary 4.1 remains true if the assumption $f: \mathbf{T} \rightarrow \mathbf{C}$ is a Dinismooth and injective function is replaced by the one that $f: \mathbf{T} \rightarrow \mathbf{C}$ is a Höldersmooth and injective function. Moreover, from (4.7) it follows that $D_{f} \leq L \pi^{\alpha} / \alpha$, and so (4.2) leads to

$$
\mathrm{L}(F) \leq \sqrt{4(L / \alpha)^{2} \pi^{2(\alpha-1)}+\|\dot{f}\|_{\infty}^{2}}
$$

Applying Theorem 3.4 we may also complement Kalaj's results; cf. [9, Thm. A, Thm. 3.1 and Cor. 3.4] and also [11].

Corollary 4.3. Let $h: \mathbf{T} \rightarrow \mathbf{C}$ be a Hölder-smooth and injective function such that $h(\mathbf{T})$ is the boundary curve of a convex domain $\Omega$ in $\mathbf{C}$. Then for every sensepreserving injective harmonic mapping $F$ of $\mathbf{D}$ onto $\Omega$ the following conditions are equivalent to each other:

(i) $F$ is a quasiconformal mapping;

(ii) $F$ is a bi-Lipschitz mapping;

(iii) $F$ has a continuous extension to the closure $\mathrm{cl}(\mathbf{D})$ and its boundary limiting valued function $f$ is absolutely continuous and satisfies the condition (3.12).

Proof. The equivalence (i) $\Longleftrightarrow$ (ii) is stated by [9, Cor. 3.4]; cf. also [11]. The equivalence (ii) $\Longleftrightarrow$ (iii) (resp. (i) $\Longleftrightarrow$ (iii)) follows from Theorem 3.4 (resp. [9, Thm. 3.1]).

\section{References}

[1] Clunie, J., and T. Sheil-Small: Harmonic univalent functions. - Ann. Acad. Sci. Fenn. Ser. A I Math. 9, 1984, 3-25.

[2] Duren, P.: Theory of $H^{p}$-spaces. - Dover Publications, Inc., Mineola, New York, 2000.

[3] Duren, P.: Harmonic mappings in the plane. - Cambridge Tracts in Math., Cambridge Univ. Press, Cambridge, 2004.

[4] FitzGerald, C. H., and F. D. Lesley: Integrability of the derivative of the Riemann mapping function for wedge domains. - J. Anal. Math. 49, 1987, 271-292.

[5] Garnett, J. B.: Bounded analytic functions. - Academic Press, New York, 1981.

[6] Heyman, W.K., and P. B. Kennedy: Subharmonic functions, volume I. - Academic Press, London, 1976.

[7] KalaJ, D.: On harmonic diffeomorphisms of the unit disc onto a covex domain. - Complex Variables 48:2, 2003, 175-187.

[8] KalaJ, D.: Quasiconformal harmonic functions between convex domains. - Publ. Inst. Math. (Beograd) (N.S.) 90, 2004, 3-20.

[9] KalaJ, D.: Quasiconformal harmonic mappings between Jordan domains. - Math. Z. 260:2, 2008, 237-252.

[10] Lewy, H.: On the non-vanishing of the Jacobian in certain one-to-one mappings. - Bull. Amer. Math. Soc. 42, 1936, 689-692.

[11] BožIn, V., M. MatelJević, and M. KnežEvić: Quasiconformality of harmonic mappings between Jordan domains. - Filomat 24:3, 2010, 111-124.

[12] Partyka, D., and K. Sakan: Quasiconformality of harmonic extensions. - J. Comput. Appl. Math. 105, 1999, 425-436. 
[13] Partyka, D., and K. SAKAn: Three variants of Schwarz's lemma for harmonic mappings. Bull. Soc. Sci. Lett. Łódź Sér. Rech. Déform. 52, 2002, 27-34.

[14] Partyka, D., and K. SAKAn: Distortion of the area measure for one-to-one harmonic mappings of the unit disk onto itself. - Sci. Bull. of Chełm, Sect. of Math. and Comp. Sci. 2, 2007, 39-48.

[15] PARTYKA, D., and K. SAKAn: On bi-Lipschitz type inequalities for quasiconformal harmonic mappings. - Ann. Acad. Sci. Fenn. Ser. A I Math. 32, 2007, 579-594.

[16] PARTYKA, D., and K. SAKAn: On a variant of Heinz's inequality for harmonic mappings of the unit disk onto bounded convex domains. Bull. Soc. Sci. Lett. Łódź Sér. Rech. Déform. 59, 2009, 25-36.

[17] PARTYKa, D., and K. SAKan: A simple deformation of quasiconformal harmonic mappings in the unit disk. - Ann. Acad. Sci. Fenn. Ser. A I Math. 37, 2012, 539-556.

[18] Pavlović, M.: Boundary correspondence under harmonic quasiconformal homeomorphisms of the unit disk. - Ann. Acad. Sci. Fenn. Ser. A I Math. 27, 2002, 365-372.

[19] Priwalow, I. I.: Randeigenschaften analytischer Funktionen. - VEB Deutscher Verlag der Wissenschaften, Berlin, 1956.

[20] Rudin, W.: Real and complex analysis. - McGraw-Hill series in higher mathematics, McGrawHill Book Company, New York, St. Louis, San Francisco, Toronto, London and Sydney, 1966.

[21] ZHU, J.-F.: Some estimates for harmonic mappings with given boundary function. - J. Math. Anal. Appl. 411, 2014, 631-638.

Received 24 October 2013 • Accepted 14 January 2014 